14

\title{
Влияние $\gamma$-облучения на характеристики деформации высоконаполненного древесно-полимерного композита
}

\author{
(C) В.В. Шпейзман ${ }^{1}$, П.Н. Якушев ${ }^{1}$, С.А. Токовой ${ }^{2,3}$, А.С. Смолянский ${ }^{2,4}$ \\ ${ }^{1}$ Физико-технический институт им. А.Ф. Иофрфе РАН, \\ Санкт-Петербург, Россия \\ ${ }^{2}$ Филиал Научно-исследовательского фризико-химического института им. Л.Я. Карпова, \\ Москва, Россия \\ ${ }^{3}$ ООО „Поливуд“, \\ Москва, Россия \\ ${ }^{4}$ Российский химико-технологический университет им. Д.И. Менделеева, \\ Москва, Россия \\ E-mail: shpeizm.v@mail.ioffe.ru
}

(Поступила в Редакцию 12 сентября 2016 г.)

С помощью прецизионной интерференционной методики измерены скорость деформации и характеристики ее скачков на микро- и наноуровне для древесно-полимерного композита, облученного до доз $0-100 \mathrm{kGy}$. Показано, что облучение приводит к упрочнению материала и к изменению характеристик скачков скорости и величины деформации. Определены размеры скачков деформации и среднеквадратичные отклонения измеренной скорости деформации от ее сглаженной зависимости от времени для скачков микро- и нанометровых размеров. Прослежено изменение этих характеристик в зависимости от дозы облучения образцов. Обнаружена связь между характеристиками микрометровых скачков и макроскопической деформацией.

Работа проведена при частичной поддержке Министерства науки и образования Российской Федерации (Субсидия от 20 октября 2014 г., № 14.576.21.0053; уникальный идентификатор прикладных научных исследований RFMEFI57614X0053) и Российского фонда фундаментальных исследований (проект № 14-07-00025).

DOI: 10.21883/FTT.2017.04.44284.347

\section{1. Введение}

Древесно-полимерные композиты (Wood-Plastic-Composites, WPC) находят широкое применение в строительстве, автомобильной и других отраслях промышленности, и их производство постоянно растет. Преимуществом этого класса материалов являются высокая технологичность, простота и дешевизна получения, экологичность. Соотношение древесины и полимера в композите может сильно изменяться в зависимости от назначения. Малое количество древесной составляющей в виде мелких частиц вводится в полимер в качестве наполнителя для придания ему большей прочности при сохранении основных достоинств полимера: малой плотности, способности к высоким, в том числе обратимым, деформациям. Композиты с большим содержанием древесины успешно конкурируют с натуральными породами дерева, существенно удешевляя и упрощая их обработку. Одним из эффективных способов воздействия на структуру материалов является их облучение электромагнитными колебаниями различной частоты и интенсивности. Известно множество работ, посвященных воздействию излучений как на полимеры и древесину в отдельности, так и на WPC (см., например, [1-5]). Исследование механических свойств этих материалов, крайне важных для их практического использования, сводилось в основном к анализу кривых растяжения, a для анизотропных материалов (древесины или WPC с древесными волокнами) дополнительно определяли прочность и максимальную деформацию при сжатии и изгибе. Меньшее внимание уделялось ползучести, несмотря на то, что при длительной эксплуатации WPC или его составляющих изменение свойств во времени приобретает первостепенное значение. На важность и необходимость изучения ползучести WPC обращалось внимание в [5].

В [6-8] описан прецизионный интерферометрический метод измерения скорости деформации при постоянной или ступенчато-возрастающей нагрузке, позволивший обнаружить разномасштабные скачки скорости и величины деформации и связать их размеры с макроскопическими характеристиками деформации материалов. В [9-11] рассмотрено влияние $\gamma$-облучения на число и размер нано- и микрометровых скачков скорости и величины деформации полиметилметакрилата (РММА) и политетрафторэтилена (РТFЕ), прослежены их изменение в зависимости от дозы облучения и связь с макроскопическими характеристиками деформации.

В $[10]$ на примере образцов РММА, предварительно подвергнутых действию $\gamma$-облучения дозами $D=0-330 \mathrm{kGy}$, показано присутствие микрометровых скачков деформации с размерами до $6 \mu \mathrm{m}$, которые имеют сложную структуру и состоят из более мелких микрометровых скачков $(1-2 \mu \mathrm{m})$. В [11] обнаружено, что малые микрометровые скачки деформации, в свою очередь, состоят из нанометровых скачков, которые 
по величине можно условно разделить на три группы: большие скачки размером 150-200 nm, средние 70-90 и малые - 10-20 nm. Облучение приводило к заметному уменьшению скорости деформации при одинаковых напряжениях и к изменению размеров скачков в зависимости от времени деформации и дозы облучения.

В [12] исследована ползучесть при ступенчатовозрастающем нагружении необлученных и облученных до $D=30 \mathrm{kGy}$ образцов PTFE при растяжении и сжатии. Кроме размера скачков, были определены среднеквадратичные отклонения скорости деформации с учетом микромасштабных скачков $S_{\overline{\Delta \dot{\varepsilon} / \varepsilon}}$ по всему интервалу деформаций в зависимости от величины напряжений, а также аналогичная зависимость для наномасштабных скачков $S_{\overline{\Delta U / U_{0}}}$ в пределах перемещения $\Delta l_{0}=325 \mathrm{~nm}$. Как было показано в [13], обе эти характеристики для карбонизированной в разных условиях древесины коррелируют с величиной неупругой деформации образцов при разрушении.

Для РТFЕ было установлено, что величина среднеквадратичного отклонения растет при переходе от режима испытаний в условиях одноосного сжатия к измеренным при растяжении, а также больше у $\gamma$-облученных образцов по сравнению с необлученными.

В настоящей работе определялись характеристики скачков скорости и величины деформации и их изменение при облучении для образцов WPC.

\section{2. Методика эксперимента}

Образцы высоконаполненных WPC с соотношением древесина/полиэтилен (РЕ) равным 80/20 испытывали при одноосном сжатии в условиях постоянных напряжений и комнатной температуре. WPC были изготовлены экструзионным методом из измельченных отходов древесины (опилки сосны) и порошка сверхвысокомолекулярного полиэтилена (UHMW PE) марки Hi-Zex Million 240M (производство Mitsui Chemicals (Япония)) со средним размером частиц $160 \mu \mathrm{m}$. В экспериментах использовали образцы цилиндрической формы с высотой 6 и диаметром $3 \mathrm{~mm}$. Облучение от источника ${ }^{60} \mathrm{Co}$ проводили на установке РХM- $\gamma-20$ в РХТУ им. Д.И. Менделеева в атмосфере воздуха при комнатной температуре. Мощность дозы составляла до $2 \mathrm{~Gy} / \mathrm{s}$, величина поглощенной дозы - от 0 до $100 \mathrm{kGy}$.

Скорость деформации $\dot{\varepsilon}$ определяли интерференционным методом, описанным в [6-13]. Расчет $\dot{\varepsilon}$ проводили по формуле $\dot{\varepsilon}=\lambda \nu / 2 l_{0}$, где $\lambda$ - длина волны источника света, $v$ - частота биений на экспериментально регистрируемой „кривой биений“, которая определялась с помощью специальной программы последовательно для каждого из биений, а $l_{0}-$ начальная длина образца. По записанной кривой ползучести $\dot{\varepsilon}(t)$ рассчитывали среднеквадратичное отклонение скорости деформации от ее значений на сглаженной кривой ползучести $S_{\overline{\Delta \dot{\varepsilon} / \varepsilon}}$ во всем интервале записанных деформаций, в которое основной вклад вносят микрометровые скачки деформации (перемещения $\Delta l$ ). Среднеквадратичное отклонение $S_{\overline{\Delta U / U_{0}}}$, представляющее собой характеристику нанометровых скачков деформации в пределах одного биения, соответствуюшего перемещению $\Delta l_{0}=325 \mathrm{~nm}$, определяли по разнице $\Delta U$ между экспериментальным значением сигнала в точках биения и его нормальным значением ( $U_{0}-$ амплитуда биения) [8]. Частота записи сигнала составляла $500 \mathrm{~Hz}$.

\section{3. Результаты опытов и их обсуждение}

На рис. 1, $a$ приведены зависимости скорости ползучести $\dot{\varepsilon}$ от времени для необлученных и облученных до различных доз образцов WPC при напряжениях $\sigma=20 \mathrm{MPa}$, а на рис. $1, b-$ кривые ползучести $\varepsilon-t$, построенные интегрированием этих зависимостей. Вид кривых на рис. 1, $a$ наглядно демонстрирует отсутствие стационарной стадии ползучести, что отмечалось нами
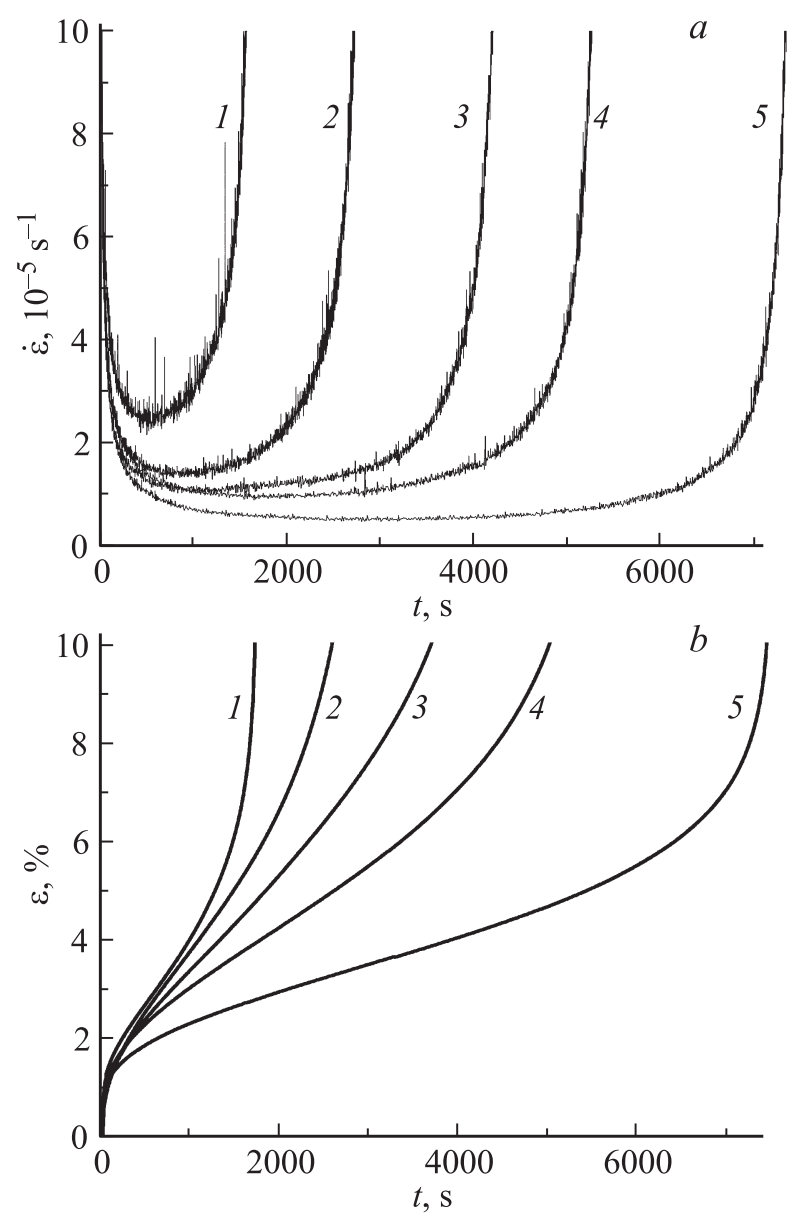

Рис. 1. Зависимость скорости $(a)$ и деформации $(b)$ от времени ползучести при одноосном сжатии для образцов WPC. Доза облучения, kGy: 0 (1), 15 (2), 30 (3), 50 (4), 100 (5). Напряжение $\sigma=20 \mathrm{MPa}$, температура $T=293 \mathrm{~K}$. 


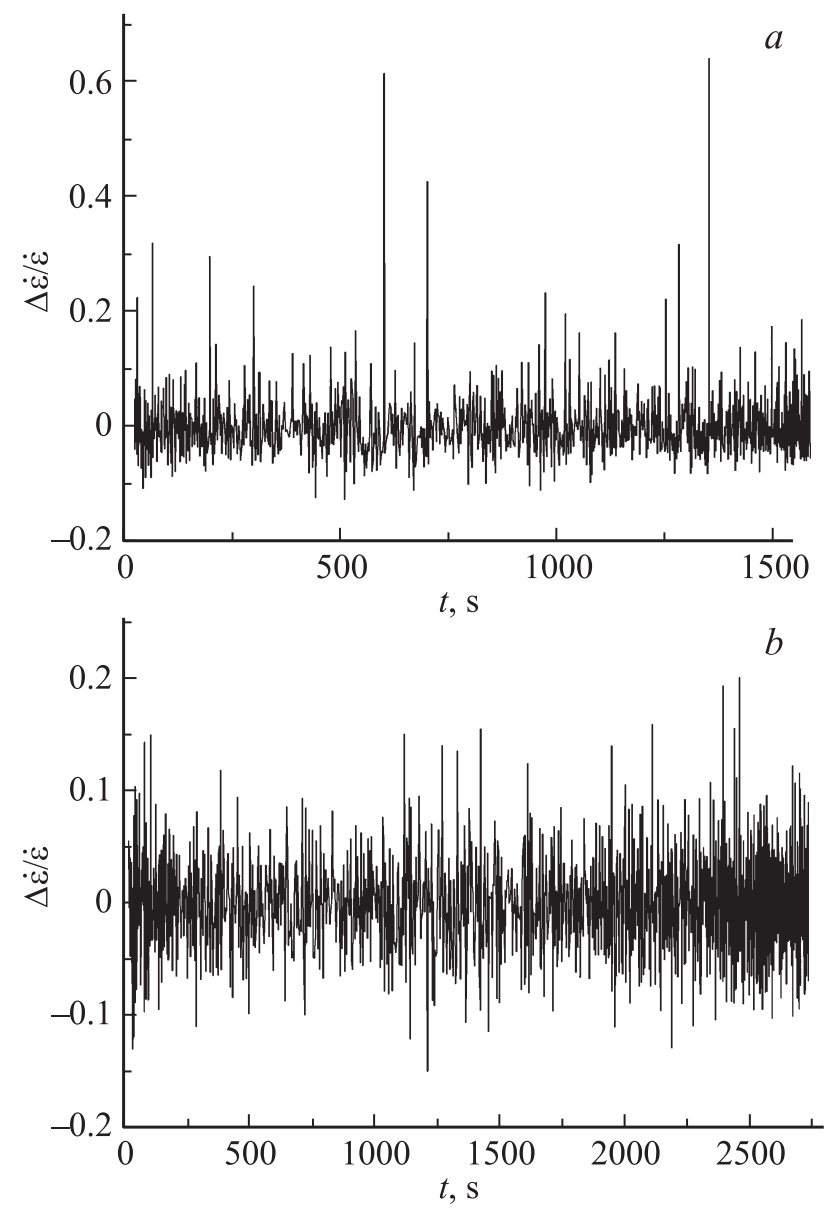

Рис. 2. Относительная величина скачка скорости деформации в зависимости от времени действия нагрузки. Доза облучения, kGy: $0(a), 15(b)$.

ранее для различных полимеров в [14]. С увеличением дозы облучения образцов наблюдается упрочнение, так как растет время, необходимое для достижения заданной деформации (рис. $1, b)$, и уменьшается скорость деформации в ее минимуме (рис. 1,a). В табл. 1 приведены значения скорости и времени деформации, соответствующие минимуму зависимости $\dot{\varepsilon}(t)$, причем время указано как абсолютное, так и в процентах от полного времени ползучести (в скобках). ${ }^{1}$ Из данных табл. 1 следует, что интервал убывания скорости (до ее минимума) меньше последующего времени возрастания скорости ползучести и составляет $28-38 \%$ полной кривой ползучести. Деформация в минимуме скорости с учетом начального скачка деформации изменяется в пределах 2.5-3.1\% при варьировании дозы облучения образцов до $100 \mathrm{kGy}$. Можно заметить, что изменение величины деформации в минимуме ее скорости при увеличении дозы облучения образцов подобно аналогичному изменению относительного времени до минимума

\footnotetext{
${ }^{1}$ Координаты минимума определялись точно по пересечению нуля производной $d \dot{\varepsilon} / d t$.
}

Таблица 1. Значения величины и скорости деформации, а также времени ползучести в точке минимума скорости в зависимости от дозы облучения образцов WPC состава 80/20

\begin{tabular}{l|c|c|c|c|c}
\hline \multicolumn{1}{c|}{ Доза, kGy } & 0 & 15 & 30 & 50 & 100 \\
\hline$t_{\min }, \mathrm{s}(\%)$ & $530(34)$ & $850(31)$ & $1160(28)$ & $1734(33)$ & $2800(38)$ \\
$\dot{\varepsilon}_{\min }, 10^{-5} \mathrm{~s}^{-1}$ & 2.50 & 1.45 & 1.12 & 0.99 & 0.55 \\
$\varepsilon_{\min }, \%$ & 2.8 & 2.6 & 2.5 & 2.7 & 3.1
\end{tabular}

скорости деформации (это следует из сравнения второй и четвертой строк табл. 1).

Переход к ускоренной ползучести означает начало процесса разупрочнения, т.е. разрушения структуры материала, которая возникла при облучении и на предыдущей стадии ползучести. Известно упрочняющее действие малых доз облучения на органические материалы [1]. Обычно этот эффект связывают с разрывом под действием облучения некоторого числа макромолекул с последующей их сшивкой, что затрудняет деформацию материала и приводит к небольшому упрочнению. Большие дозы облучения создают сильно искаженную структуру, образуются трещины и поры, и вследствие этого прочность материала падает. В нашем случае время до разрушения (резкого возрастания скорости деформации), а также абсолютное значение времени, соответствующее минимуму скорости, возрастают, а значение скорости деформации в ее минимуме уменьшается с ростом дозы облучения, что свидетельствует об упрочнении материала при облучении. Рост деформации и относительного времени в минимуме кривой $\dot{\varepsilon}(t)$ при 50 и $100 \mathrm{kGy}$ можно связать с различными пороговыми значениями дозы облучения, при которой начинаются разрывы макромолекул, для полимера и древесины.

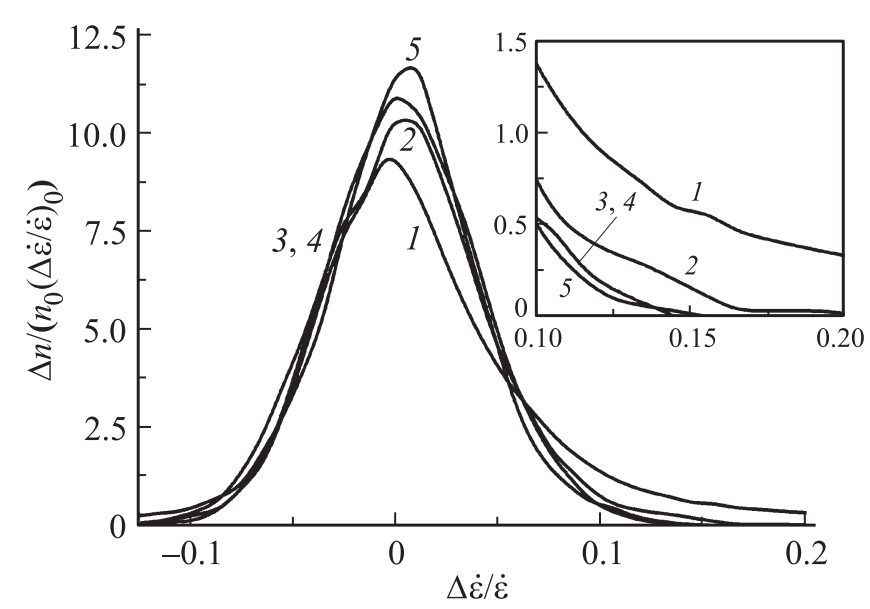

Рис. 3. Кривые распределения скачков скорости $\Delta \dot{\varepsilon} / \dot{\varepsilon}$ по размерам: $n_{0}-$ общее число измеренных микрометровых скачков, $\Delta n$ - число скачков в выбранном интервале $(\Delta \dot{\varepsilon} / \dot{\varepsilon})_{0}=0.1$. Отдельно показано распределение в области больших скачков c $\Delta \dot{\varepsilon}>0$. Доза облучения, kGy: 0 (1), 15 (2), 30 (3), 50 (4), $100(5)$. 
Таблица 2. Характеристики скачков деформации в микро- и нанометровом диапазонах в зависимости от дозы облучения образцов WPC

\begin{tabular}{c|c|c|c|c|c}
\hline Доза, kGy & 0 & 15 & 30 & 50 & 100 \\
\hline$S_{\overline{\Delta \dot{\varepsilon} / \dot{\varepsilon}}}$ & 0.0581 & 0.0458 & 0.0412 & 0.0409 & 0.0415 \\
$S_{\overline{\Delta \dot{\varepsilon} / \dot{\varepsilon}} \text { left }}$ & 0.0555 & 0.0441 & 0.0411 & 0.0395 & 0.0396 \\
$S_{\overline{\Delta \dot{\varepsilon} / \dot{\varepsilon}} \text { right }}$ & 0.0592 & 0.0464 & 0.0413 & 0.0422 & 0.0423 \\
$S_{\overline{\Delta U / U_{0}}}$ & 0.1077 & 0.1183 & 0.1473 & 0.1407 & 0.1695 \\
$L, \mu \mathrm{m}$ & $1.4 \pm 0.5$ & $1.2 \pm 0.4$ & $1.0 \pm 0.4$ & $1.0 \pm 0.3$ & $1.1 \pm 0.3$ \\
& $13.5 \pm 3.7$ & $11.0 \pm 3.9$ & $10.6 \pm 4.6$ & $11.4 \pm 3.3$ & $12.7 \pm 4.1$ \\
$L, \mathrm{~nm}$ & $\sim 1$ (мало́) & $0.75 \pm 0.14$ & $0.69 \pm 0.11$ & $0.47 \pm 0.11$ & $0.32 \pm 0.18$ \\
& $5.9 \pm 0.5$ & $\sim 4$ (мало́) & $\sim 4$ (мало́) & $\sim 4$ (мало́) & $3.0 \pm 0.7$ \\
& $\sim 20$ (мало́) & $21.3 \pm 7.2$ & $19.8 \pm 9.0$ & $26.7 \pm 5.3$ & $24.9 \pm 5.7$ \\
& $112.4 \pm 20.7$ & $\sim 100$ (мало́) & $\sim 100$ (мало́) & $\sim 100$ (мало́) & $\sim 100$ (мало́)
\end{tabular}

Известно, что пороговые значения дозы облучения, при которой начинается разупрочнение материала при сжатии, для полимера и древесины различны. Так, полиэтилен, входящий в состав исследованного нами WPC, обладает радиационной стойкостью $10^{2}-10^{3} \mathrm{kGy}$ [1]. По данным [3], при облучении до доз $1 \mathrm{MGy}$ прочность древесины практически не изменяется, в то время как при $5 \mathrm{MGy}$ прочность при сжатии снижается на 30\%. Скорость ползучести - значительно более чувствительная характеристика, чем величина прочности, поэтому влияние облучения на нее может проявляться при меньших дозах. Отмеченная выше немонотонность
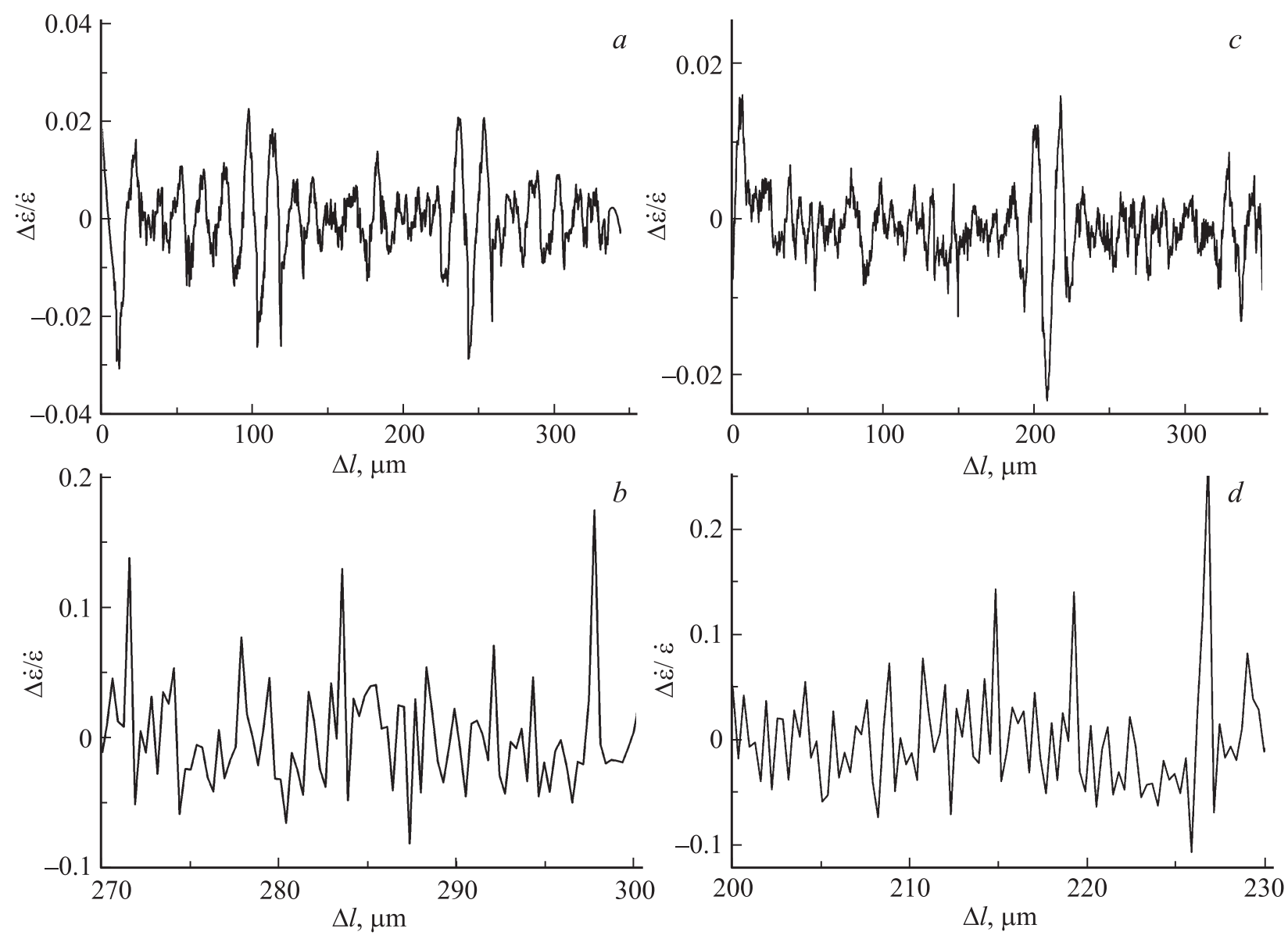

Рис. 4. Сглаженные $(a, c)$ и измеренные $(b, d)$ зависимости относительной величины скачка скорости деформации от изменения длины образца. Доза облучения, $\mathrm{kGy:} 0(a, b), 50(c, d)$. 

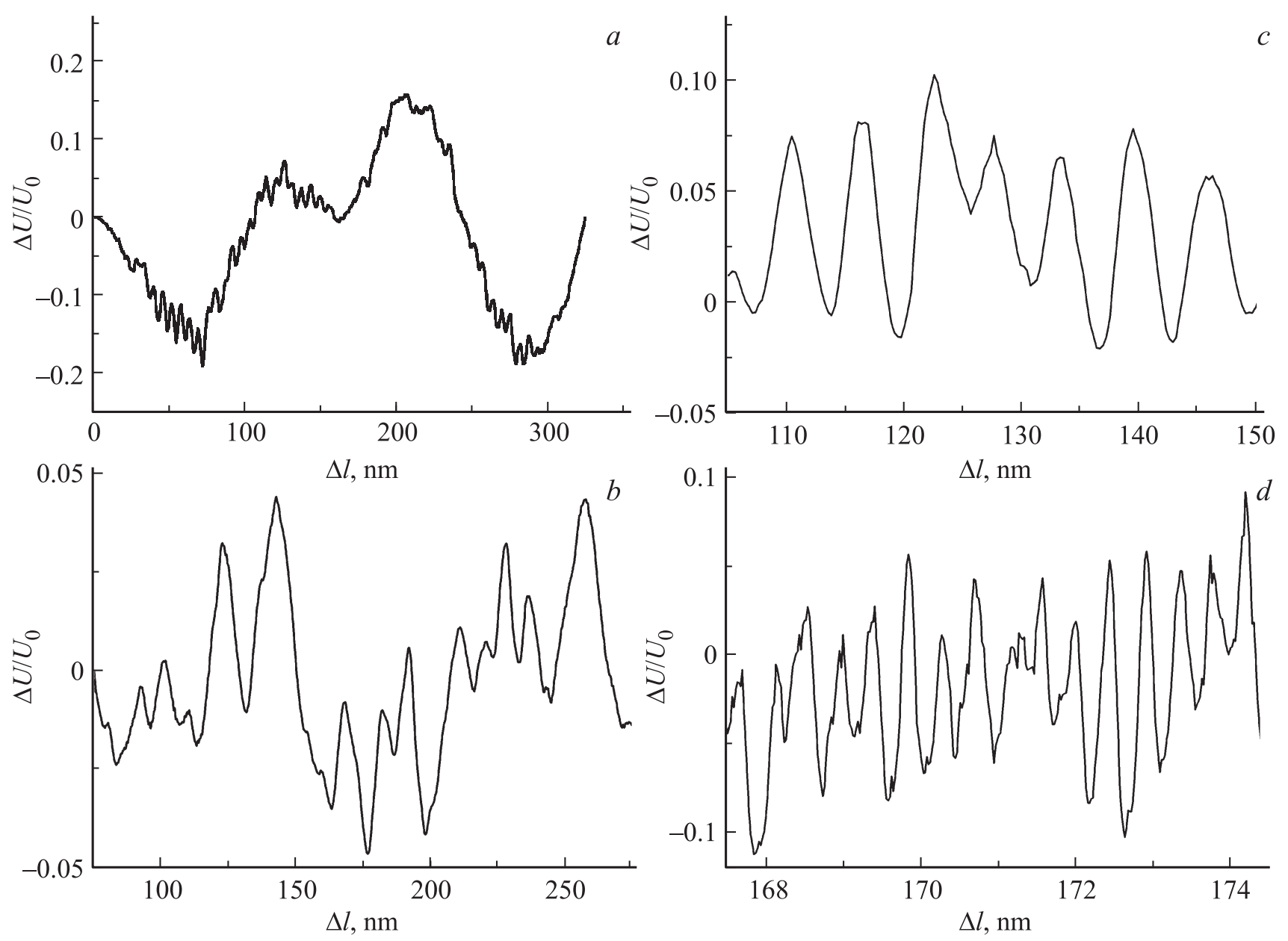

Рис. 5. Примеры нанометровых скачков деформации разного размера. Доза облучения, kGy: 0 (a,c), $30(b), 50(d)$. Средние размеры скачков деформации, $\mathrm{nm}: 110(a), 20(b), 6(c), 0.5(d)$.

зависимости критической деформации и относительного времени перехода от упрочнения к разупрочнению с ростом дозы облучения может быть вызвана тем, что при величине поглощенной дозы до $\sim 50 \mathrm{kGy}$ эффект связан с изменением структуры только полимера. В области доз от 50 до $100 \mathrm{kGy}$ происходит согласованное изменение структуры древесины и полимера, и доля неопасной затухающей ползучести возрастает.

Высокая точность измерения скорости деформации интерференционным методом на базе линейных перемещений $\Delta l_{0}=325 \mathrm{~nm}$ позволила наблюдать неоднородность (скачки) скорости и величины деформации. Скачки скорости деформации хорошо видны на ее зависимости от времени (рис. 1,a). Однако кривая ползучести $\varepsilon(t)$ выглядит плавной из-за малости $\Delta l_{0}$ (рис. $1, b)$. На рис. 2 приведены временные зависимости относительного изменения скорости деформации $\Delta \dot{\varepsilon} / \dot{\varepsilon}$, где $\Delta \dot{\varepsilon}-$ отклонение измеренной скорости деформации от ее значения на плавной сглаженной кривой, для необлученных и облученных до $15 \mathrm{kGy}$ образцов WPC. Видно, что кратковременные относительные ускорения деформации больше, чем замедления, особенно для не- облученных образцов. Максимальные скачки скорости деформации необлученных образцов $\Delta \dot{\varepsilon} / \dot{\varepsilon}$ достигают величины 0.5 , в то время как для облученных до $15 \mathrm{kGy}$ образцов $\Delta \dot{\varepsilon} / \dot{\varepsilon}$ не превышает 0.2. Увеличение дозы облучения до $100 \mathrm{kGy}$ не приводит к заметному изменению вида кривых $\Delta \dot{\varepsilon} / \dot{\varepsilon}(\Delta l)$. В табл. 2 приведены среднеквадратичные отклонения относительного изменения скорости деформации $S_{\overline{\Delta \dot{\varepsilon} / \varepsilon}}$ для всех испытанных образцов.

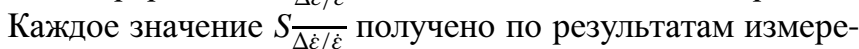
ний $\sim 1300$ значений $\Delta \dot{\varepsilon} / \dot{\varepsilon}$. Как видно из таблицы, $S_{\overline{\Delta \dot{\varepsilon} / \dot{\varepsilon}}}$ уменьшается при облучении до дозы $30 \mathrm{kGy}$, а дальше остается примерно постоянной. Это изменение связано с поведением больших положительных скачков $\Delta \dot{\varepsilon} / \dot{\varepsilon}$, т. е. с правым крылом распределений $\Delta \dot{\varepsilon} / \dot{\varepsilon}$, которые приведены на рис. 3. Максимумы распределения слегка сдвинуты вправо от нуля, а кривые в области больших отклонений для необлученных образцов идут выше остальных. Ниже располагается кривая для образцов, облученных до $15 \mathrm{kGy}$, еще ниже - все остальные, разница между которыми невелика. Количественно этот эффект можно оценить, сравнивая среднеквадратичные отклонения для 
отрицательных $\left(S_{\overline{\Delta \dot{\varepsilon} / \varepsilon}}\right.$ left $)$ и положительных $\left(S_{\overline{\Delta \dot{\varepsilon} / \varepsilon}}\right.$ right $)$ скачков скорости деформации (см. табл. 2). ${ }^{2}$

Величину скачков деформации (осевого перемещения при сжатии) предложено оценивать по расстоянию между близкими по размеру скачками скорости деформации на зависимости $\dot{\varepsilon}(\Delta l)$ или $\Delta \dot{\varepsilon} / \dot{\varepsilon}(\Delta l)[8-13]$. Используя экспериментальные (растянутые малые участки кривых, подобных изображенным на рис. 2) и сглаженные зависимости $\Delta \dot{\varepsilon} / \dot{\varepsilon}(\Delta l)$, можно выделить характерные размеры $\Delta l$ в микрометровой области. На рис. 4 показаны участки зависимости $\Delta \dot{\varepsilon} / \dot{\varepsilon}(\Delta l)$, построенные по экспериментальным точкам при изменении длины образца $\Delta l=30 \mu \mathrm{m}$, и участки сглаженной кривой с $\Delta l=350 \mu \mathrm{m}$ для необлученного и облученного до $50 \mathrm{kGy}$ образца WPC. Анализ размеров скачков деформации по расстояниям между всеми соседними пиками на рис. $4, a, b$ показывает, что их можно разделить на две группы: с размерами около $1-2$ и $10-15 \mu \mathrm{m}$. Средняя величина скачка для необлученного WPC составляет $1.4 \pm 0.5$ и $13.5 \pm 3.7 \mu$ m для малых (микрометровых) и для более крупных скачков соответственно. На рис. $4, b$ видны два больших интервала $(15.7$ и $11.5 \mathrm{~nm})$, что хорошо совпадает с размером скачка, определенным по сглаженной кривой (рис. $4, a$ ). Размеры скачков деформации для облученных образцов приведены в табл. 2. В зависимости от дозы облучения наблюдается зависимость, близкая к описанной выше для среднеквадратичного отклонения скорости деформации: при малых дозах размер скачков уменьшается, а затем остается примерно постоянным.

Оценка характеристик нанометровых скачков скорости и величины деформации проводилась по выбранным биениям на экспериментально зарегистрированной „кривой биений“ вблизи минимума скорости деформации [8]. Для нанометровых скачков наблюдаются большие значения среднеквадратичных отклонений скорости по сравнению с микрометровыми скачками (см. табл. 2), что, возможно, определяется более высокой степенью локализации деформации. Не исключено также влияние уровня шума на величину сигнала $U$. Направление изменения $S_{\overline{\Delta U / U_{0}}}$ с ростом дозы облучения оказалось противоположным рассмотренному выше для микрометровых скачков скорости: $S_{\overline{\Delta U / U_{0}}}$ растет с увеличением дозы. Скачки величины сигнала $\Delta U / U_{0}$ для облученных образцов можно разделить на три группы: большие (десятки $\mathrm{nm}$ ), средние (единицы $\mathrm{nm}$ ) и малые $(1 \mathrm{~nm}$ и менее). Для необлученных образцов наблюдались также скачки деформации размером более $100 \mathrm{~nm}$. На рис. 5 приведены примеры скачков для облученных и необлученных образцов, а в табл. 2 указаны средние размеры часто встречающихся скачков.

\footnotetext{
2 Заметим, что если принять отмеченный выше факт расположения минимума скорости вблизи 1/3 полного времени испытания, то значения приведенных в табл. 1 значений среднеквадратичных отклонений не являются независимыми, а связаны соотношением: $S_{\overline{\Delta \dot{\varepsilon} / \dot{\varepsilon}}}=\left(1 / 3\left(S_{\overline{\Delta \dot{\varepsilon} / \dot{\varepsilon}}} \text { left }\right)^{2}+2 / 3\left(S_{\overline{\Delta \dot{\varepsilon} / \dot{\varepsilon}}} \text { right }\right)^{2}\right)^{0.5}$, в чем можно убедиться, используя приведенные в табл. 2 числа.
}

Облучение приводит к уменьшению размеров скачков. Практически пропадают очень крупные скачки, которые наблюдались в необлученных образцах, и появляются очень мелкие скачки. С увеличением дозы облучения наблюдается тенденция к уменьшению размера скачков и появлению очень мелких скачков деформации. Полученные результаты подтверждают предположение о том, что разрушение под действием облучения цепных молекул полимера и дерева приводит к уменьшению размеров частиц, согласованное движение которых обеспечивает деформацию на разных ее уровнях.

\section{4. Заключение}

Использование прецизионной методики измерения скорости деформации позволяет получить новые характеристики процесса на разных его уровнях. В настоящей работе изучено влияние $\gamma$-облучения на скорость ползучести образцов WPC и характеристики ее скачков на микро- и наноуровне. Наличие минимума на зависимости скорости ползучести от времени позволило сопоставить изменение его координат и деформации в минимуме для образцов, подвергнутых облучению до доз $0-100 \mathrm{kGy}$. Рассчитано среднеквадратичное отклонение скорости деформации от ее значений на сглаженной зависимости скорости от деформации в микро- и нанометровом диапазонах. Полученные данные позволяют описать деформацию WPC как многоуровневый процесс с преобладанием двух микрометровых $(1-2$ и $10-15 \mu \mathrm{m})$ и двух (для доз $0-50 \mathrm{kGy}$ ) и трех (для $100 \mathrm{kGy}$ ) нанометровых (от $<1$ до $>100 \mathrm{~nm})$ характерных размеров элементарных перемещений (скачков деформации). Микрометровые скачки деформации необлученных образцов меньше, чем облученных. Так же себя ведет среднеквадратичное отклонение $S_{\overline{\Delta \dot{\varepsilon} / \varepsilon}}$ и величина скорости деформации в ее минимуме. У нанометровых скачков всего обнаружено четыре подуровня. Среднеквадратичное отклонение $S_{\overline{\Delta U / U_{0}}}$ растет с ростом дозы облучения образцов, что, возможно, связано с уменьшением скорости деформации в ее минимуме при росте дозы облучения. Подобная зависимость $S_{\overline{\Delta U / U_{0}}}$ от скорости деформации наблюдалась в [14] для РТFE. Роль нанометровых скачков, по-видимому, заключается в формировании скачков следующего уровня - микрометровых, которые образуют макроскопическую деформацию образца. Изменение характеристик микрометровых скачков деформации (размера и среднеквадратичного отклонения скорости) при облучении образцов хорошо соответствует изменению деформации перехода к ускоренной ползучести, что подтверждает предположение о существовании взаимосвязи микрометровых скачков скорости и величины деформации с макроскопической деформацией образца. 


\section{Список литературы}

[1] В.К. Милинчук, Э.Р. Клиншпонт, В.И. Тупиков. Основы радиационной стойкости органических материалов. Энергоатомиздат, М. (1994). 256 с.

[2] В.С. Иванов. Радиационная химия полимеров. Химия, Л. (1988). $320 \mathrm{c}$

[3] А.С. Фрейдин. Действие ионизирующей радиации на древесину и ее компоненты. Гослесбумиздат, М.-Л. (1961). $119 \mathrm{c}$.

[4] D. Ndiaye, A.M. Badji, A. Tidjani. J. Comp. Mater. (2013). http://jcm.sagepub.com/content/early/2013/10/11/ 0021998313506241

[5] A. Palm, J. Smith, M. Driscoll, L. Smith, L.S. Larsen. Phys. Procedia 66, 595 ( 2015).

[6] Н.Н. Песчанская. Высокомолекуляр. соединения А 31, 1181 (1989).

[7] Н.Н. Песчанская, П.Н. Якушев. ФТТ 40, 9, 1635 (1998).

[8] В.В. Шпейзман, Н.Н. Песчанская. ФТТ 51, 6, 1087 (2009).

[9] Н.Н. Песчанская, В.В. Шпейзман, П.Н. Якушев, А.С. Смолянский, А.С. Шведов. Изв. РАН. Сер. физ. 73, 10, 1514 (2009).

[10] Н.Н. Песчанская, А.С. Смолянский, А.С. Шведов. ФТТ 51, 6, 1218 (2009)

[11] В.В. Шпейзман, Н.Н. Песчанская, П.Н. Якушев, А.С. Смолянский, А.С. Шведов, В.Г. Черемисов. ФТТ 52, 2, 248 (2010).

[12] В.В. Шпейзман, П.Н. Якушев, Л.И. Трахтенберг, А.С. Смолянский. ФТТ 56, 12, 2399 (2014).

[13] В.В. Шпейзман, Т.С. Орлова, Б.К. Кардашев, Б.И. Смирнов, A. Gutierrez-Pardo, J. Ramirez-Rico. ФТT 56, 522 (2014).

[14] В.В. Шпейзман, П.Н. Якушев, Н.Н. Песчанская, Ж.В. Мухина, А.С. Шведов, В.Г. Черемисов, А.С. Смолянский. ФТТ 54, 6, 1149 (2012). 